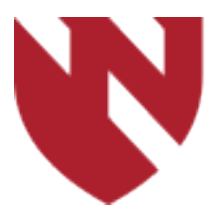

September 2020

\title{
Authorship - Perspective of an ENT Resident
}

Matthew T. Solverson

University of Nebraska Medical Center

Tell us how you used this information in this short survey.

Follow this and additional works at: https://digitalcommons.unmc.edu/gmerj

Part of the Bioethics and Medical Ethics Commons, Higher Education Commons, Interprofessional Education Commons, and the Medical Humanities Commons

\section{Recommended Citation}

Solverson, M. T. Authorship - Perspective of an ENT Resident. Graduate Medical Education Research Journal. 2020 Sep 29; 2(1). https://digitalcommons.unmc.edu/gmerj/vol2/iss1/2 


\title{
Authorship - Perspective of an ENT Resident
}

\author{
Abstract \\ N/A due to perspective piece \\ Keywords \\ authorship, publication, research \\ Creative Commons License \\ (c) $($ i) $\Theta \Theta$
}

This work is licensed under a Creative Commons Attribution-Noncommercial-No Derivative Works 4.0 License.

\section{Cover Page Footnote}

A special thank you to Dr. Jayme Dowdall for her support and guidance in this process. 


\section{Authorship - Perspective of an ENT Resident \\ Matt Solverson}

University of Nebraska Medical Center, Department of Otolaryngology - Head and Neck Surgery

Academic research is a major contributor to the advancement of medical practice. A published manuscript is the medium through which research is conveyed to the medical community. Currently, single-author publications are becoming increasingly rare, whereas large, multicenter trials and their associated publications are increasingly prevalent. ${ }^{1}$ The concept of authorship is frequently considered but less frequently discussed. The details of authorship have become increasingly complex with the movement towards team-based research.

A large percentage of prospective and active residents are involved in research during their training, and many of their prospective employers prioritize research experience. Specifically for students, publications have been associated with increased odds of matching to surgical specialties. ${ }^{2,3}$ For residents seeking fellowship, priority is often given to applicants with a strong history of publications in peer-reviewed journals. The importance of research publications in applying to surgical fellowship is highlighted by Patel and colleagues, ${ }^{4}$ who demonstrated that more than $10 \%$ of orthopedic surgery fellowship applicants misrepresented their citations to bolster their candidacy. For trainees attempting to match into such residency and fellowship positions, the ethical standards of authorship have clear importance.

Inappropriate declaration of authorship has led to terms such as "ghost-authorship" and "gift-authorship." The former refers to a person who contributed significantly, but was not formally recognized, whereas the latter refers to a person formally recognized despite lack of significant contribution to a scholarly project. ${ }^{5}$ The International Committee of Medical Journal Editors (ICMJE) proposed a set of criteria to combat such practices. Currently, four criteria are recommended for a person to be included as an author:

1) Significant contributions to concept or design; acquisition, analysis, or interpretation of data; AND 2) Drafting or critically editing the work for important intellectual content; AND 3) Approving the final version to be published; AND 4) Agreeing to be accountable for all aspects of the work in ensuring that questions related to the accuracy or integrity of any part of the work are appropriately investigated and resolved. ${ }^{6}$
Despite these criteria, problems with authorship continue to exist but are rarely discussed. In a Letter to the Editor, Lypson and Phelbert ${ }^{7}$ call on the academic community to recognize such issues and advocate for the maintenance of a high ethical standard for authorship. The letter highlights an example of research in which two medical students were initially omitted from the author list but were later formally included through an erratum. ${ }^{8}$ Additionally, Karani and colleagues 5 reviewed medical students' experience with authorship during an NIH-funded research experience, noting only two-thirds of students indicated they clearly discussed authorship criteria and expectations during the process, and less than one-third of students indicated having prior formal training on authorship. These statistics highlight a need for academic leaders to more openly discuss authorship and teach the ICMJE guidelines to trainees.

Teaching ethical authorship to medical trainees may be a logical next-step in addressing authorship issues, however multiple dilemmas remain. No guidelines have been advanced regarding the discussion of authorship expectations. The ICMJE loosely advises that authorship discussion should occur during the planning of the work, and that appropriate modifications be made as the work progresses. ${ }^{6}$ Students and residents may represent a vulnerable population in these discussions, given the nature of the relationship between trainees and their research supervisors. Karani and colleagues 5 noted that, of those medical students who indicated having concerns about authorship, over half did not raise their concerns due to fear of challenging their mentor. Research supervisors often have a position of authority over trainees, and thus the ability to influence trainees' career prospects. This power dynamic may make trainees reluctant to raise concerns about authorship.

Additionally, it is unclear who should retain the responsibility to ensure authors meet ICMJE criteria. Some journals now require each self-reported author to specify their contributions and/or formally attest they have met authorship criteria. However, many argue that journals and editors are not equipped to determine who should be included as authors, rather that it is the responsibility of the authors themselves to determine who meets criteria..$^{9,10}$ Unfortunately, self-determination of authorship has the potential to introduce dishonesty or bias in the deciding which contributions may be considered substantial. Similarly, concepts such as "guest-authorship" arise, in which an individual with name recognition is included as an author to enhance the likelihood of publication. ${ }^{11}$ Such challenges to ethical authorship threaten the value of academic research. Unfortunately, there is not a simple solution to prevent similar improprieties.

Transparency and accountability are key tenets of ethical authorship. The ICMJE guidelines provide clear recommendations which we hope trainees can use to remove barriers to discussing authorship up-front with their collaborators and empower them to claim rightful authorship when deserving. It is the duty of academic leaders to teach these guidelines, but all members of the medical community have the responsibility to enforce authorship integrity. This helps to confer the proper respect to academic research and engender public trust in medical advancement through research.

https://doi.org/10.32873/unmc.dc.gmerj.2.1.006

\section{References}

1 Fontanarosa P, Bauchner H, Flanagin A. Authorship and team science. JAMA. 2017;318(24):2433-2437.

2 Borsting EA, Chim JH, Thaller SR. An updated view of the integrated plastic surgery match. Ann Plast Surg. 2015;75:556-559.

3 Hauser LJ, Gebhard GM, Blumhagen R, et al. Applicant characteristics associated with successful matching into otolaryngology. Laryngoscope. 2017 May;127(5):1052-1057.

4 Patel MV, Pradhan BB, Meals RA. Misrepresentation of research publications among orthopedic surgery fellowship applicants: a comparison with documented misrepresentations in other fields. Spine (Phila $\mathrm{Pa}$ 1986). 2003 Apr 1;28(7):632-36.

5 Karani R, Ognibene FP, Fallar R, et al. Medical students' experiences with authorship in biomedical research. Aca Med. 2013;88(3):364-368.

6 International Committee of Medical Journal Editors. Recommendations for the Conduct, Reporting, Editing, and Publication of Scholarly Work in Medical Journals. Updated December 2019. http://www.icmje. org/icmje-recommendations.pdf. Accessed December $15,2019$.

7 Lypson ML, Philibert I. An ethical argument for preserving medical trainee authorship. Academic Medicine. 2013;88(10):1404.

8 Rinard R, Garol BD, Shenoy AB, et al. Successfully matching into surgical specialties: An analysis of national resident matching program data. J Grad Med Educ. 2010;2:316-321.

9 Elston DM. Authorship. J Am Acad Derm. 2018 Dec;79(6):1025.

10 Flanagan J. Editorial-Authorship. Int'l J of Nursing Knowledge. 2016 Jan 28;27(1):3.

11 Citrome L. Authorship: Musings about guests and ghosts. The Int'l J of Med Prac. 2017 July 25;71(7). 